Document downloaded from:

http://hdl.handle.net/10251/166756

This paper must be cited as:

Cassiraga, EF.; Gómez-Hernández, JJ.; Berenguer, M.; Sempere-Torres, D.; Rodrigo-Ilarri, J. (2021). Spatiotemporal Precipitation Estimation from Rain Gauges and Meteorological Radar Using Geostatistics. Mathematical Geosciences. 53(4):499-516. https://doi.org/10.1007/s11004-020-09882-1

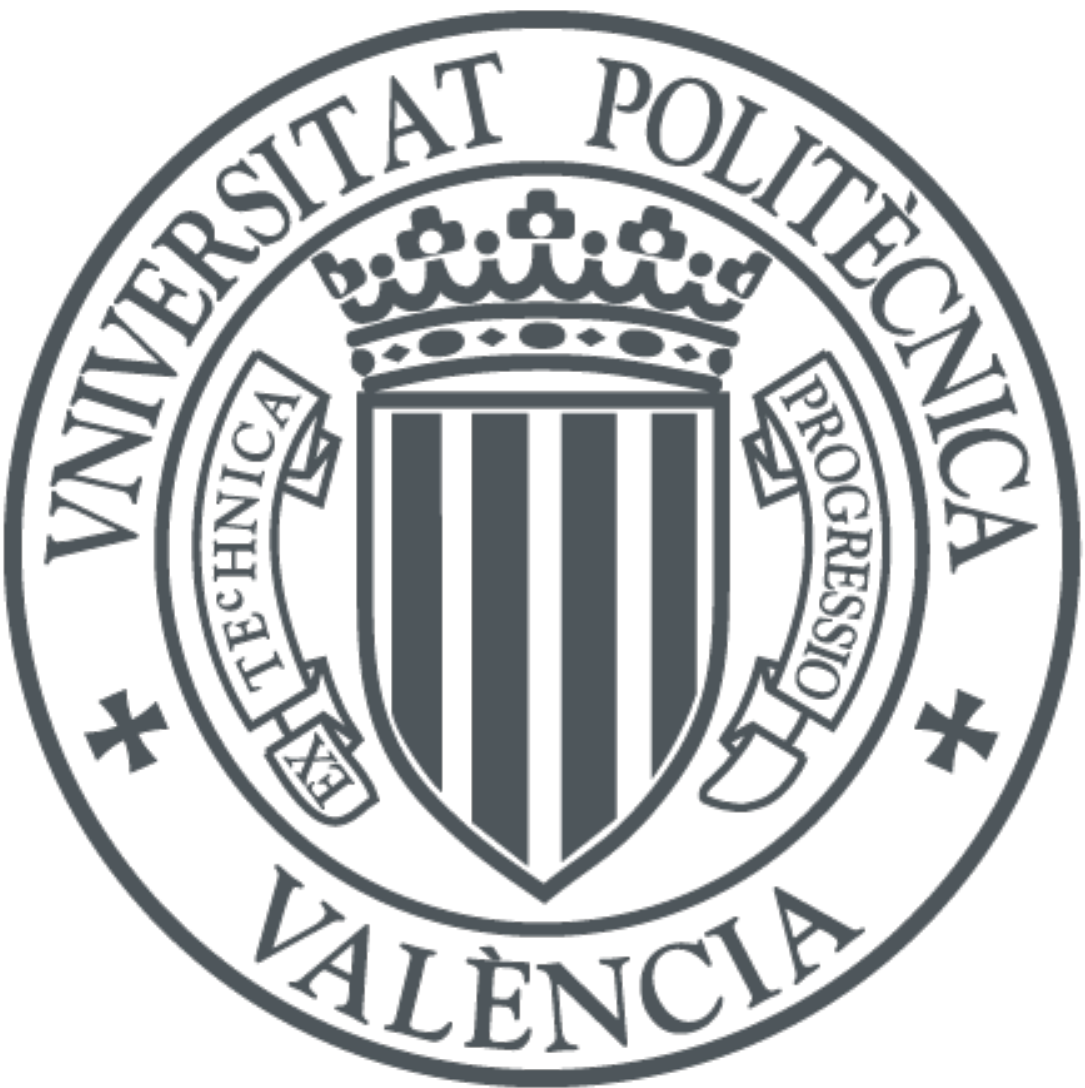

The final publication is available at

https://doi.org/10.1007/s11004-020-09882-1

Copyright Springer-Verlag

Additional Information 


\title{
Spatiotemporal Precipitation Estimation from Rain Gauges and Meteorological Radar Using Geostatistics
}

\author{
Eduardo Cassiraga • \\ J. Jaime Gómez-Hernández . \\ Marc Berenguer • Daniel Sempere-Torres . \\ Javier Rodrigo-Ilarri
}

Received: date / Accepted: date

\begin{abstract}
Automatic interpolation of precipitation maps combining rain gauge and radar data has been done in the past but considering only the data collected at a given time interval. Since radar and rain gauge data are collected at short intervals, a natural extension of previous works is to account for temporal correlations and to include time into the interpolation process. In this work, rainfall is interpolated using data from the current time interval and the previous one. Interpolation is carried out using kriging with external drift, in which the radar rainfall estimate is the drift, and the mean precipitation is set to zero at the locations where the radar estimate is zero. The rainfall covariance is modeled as non-stationaary in time, and the space system of reference moves with the storm. This movement serves to maximize the collocated correlation between consecutive time intervals. The proposed approach is analyzed for four episodes that took place in Catalonia (Spain). It is compared with three other approaches: (i) radar estimation, (ii) kriging with external drift using only the data from the same time

Eduardo Cassiraga

Institute of Water and Environmental Engineering, Universitat Politècnica de València, Valencia, Spain

E-mail: efc@dihma.upv.es

J. Jaime Gómez-Hernández

Institute of Water and Environmental Engineering, Universitat Politècnica de València, Valencia, Spain

E-mail: jgomez@upv.es

Marc Berenguer

Center of Applied Research in Hydrometeorology, Universitat Politècnica de Catalunya, Barcelona, Spain

E-mail: marc.berenguer@crahi.upc.edu

Daniel Sempere-Torres

Center of Applied Research in Hydrometeorology, Universitat Politècnica de Catalunya, Barcelona, Spain

E-mail: sempere@crahi.upc.edu

Javier Rodrigo-Ilarri

Institute of Water and Environmental Engineering, Universitat Politècnica de València, Valencia, Spain

E-mail: jrodrigo@upv.es
\end{abstract}


interval, and (iii) kriging with external drift using data from two consecutive time intervals but not accounting for the displacement of the storm. The comparisons are performed using cross-validation. In all four episodes, the proposed approach outperforms the other three approaches. It is important to account for temporal correlation and use a Lagrangian system of coordinates that tracks the rainfall movement.

Keywords rain interpolation - space-time modeling · Lagrangian extrapolation · automatic modeling

\section{Introduction}

Precipitation data are the primary input to hydrological modeling. Rain gauge networks and meteorological radars are commonly used to measure precipitation. Rain gauges collect point measurements (with a sampling size of about $200 \mathrm{~cm}^{2}$ ) irregularly in space and with a low spatial density. Weather radar measures precipitation remotely and indirectly but with a high spatiotemporal resolution (one observation per square kilometer and every 5 to 10 minutes). The low density of the rain gauge networks prevents an accurate characterization of the spatial variability of rainfall. The radar rainfall estimates can improve such a characterization, but they are prone to systematic and local errors that must be filtered out before its usage for hydrological purposes.

Radar rainfall estimates have proven very valuable in the spatial estimation of rainfall, even when rain gauge networks are dense (Sempere-Torres et al. 1999; Seo 1998; Sun et al. 2000). There are several implementations for the spatial estimation of rainfall combining rain gauges and radar observations. They use different techniques and algorithms, such as the simple calibration of multiplicative factors (Chumchean et al. 2006; Harrold and Austin 1974; Wilson and Brandes 1979), multivariate statistical analysis (Brown et al. 2001; Hevesi et al. 1992a,b), probabilistic analysis of the joint distribution of rain gauge and radar observations (Calheiros and Zawadzki 1987; Rosenfeld et al. 1993, 1994, 1995), geostatistics (Azimi-Zonooz et al. 1989; Creutin et al. 1988; Delrieu et al. 2014; Goudenhoofdt and Delobbe 2009; Jewell and Gaussiat 2015; Krajewski 1987; Seo 1998; Seo et al. 1990; Sideris et al. 2014; Sinclair and Pegram 2005; Velasco-Forero et al. 2009; Yoon and Bae 2013), or Bayesian analysis (Todini 2001). More recently, a few researchers have attempted to incorporate the temporal component into the analysis Pulkkinen et al. (2016) or Sideris et al. (2014).

This work aims to present a methodology based on geostatistics capable of estimating rain fields from rain gauge and radar data collected at two consecutive time intervals. Kriging with external drift is the geostatistical technique chosen with the following considerations: (i) the mean rainfall is set to zero if the radar observation is zero, (ii) the rainfall covariance is modeled as non-stationary in time, and (iii) the movement of the rainfall field between time steps is incorporated into the estimation process by using a Lagrangian system of reference. This methodology is a step forward in the line of work started by Velasco-Forero et al. (2009), who implemented a technique for the automatic estimation of rainfall fields in space with the data collected at a single time interval.

In the following, kriging with external drift, as applied by Velasco-Forero et al. (2009), is presented. A modified version incorporating data from two consecutive 
time steps and accounting for a non-stationary covariance is described next. The explanation on how to account for the motion of the rainfall field in the estimation process follows. The description of the data set, the four case studies, and the discussion of the results of the different estimation alternatives end the paper.

\section{Theory}

\subsection{Kriging with External Drift for a Given Time Step}

Kriging with external drift (KED) was used by Velasco-Forero et al. (2009) to estimate rainfall by merging radar and rain gauge data accumulated during the same time interval. The standard formulation of KED, as presented, for instance, in Journel and Rossi (1989) or Deutsch (1991), assumes that precipitation is a regionalized variable $z(\mathbf{x})$ modeled by a random function $Z(\mathbf{x})$, the expected value of which $m(\mathbf{x})$ is non-stationary and depends linearly on a known drift function $R(\mathbf{x})$ according to the following expression

$$
m(\mathbf{x})=a+b \cdot R(\mathbf{x}),
$$

with unknown parameters $a$ and $b$.

Under this assumption, the kriging estimate of $z$ at location $\mathbf{x}_{0}$ is a linear function of the $n\left(\mathbf{x}_{0}\right)$ surrounding values falling within a predefined ellipse around $\mathbf{x}_{0}$ given by

$$
z_{K E D}^{*}\left(\mathbf{x}_{0}\right)=\sum_{i=1}^{n\left(\mathbf{x}_{0}\right)} \lambda_{i}(\mathbf{x}) z\left(\mathbf{x}_{i}\right),
$$

with weights $\lambda_{i}$ obtained from the solution of the system of linear equations

$$
\left(\begin{array}{ccccc}
C_{11} & \cdots & C_{1 n} & 1 & R_{1} \\
\vdots & \ddots & \vdots & \vdots & \vdots \\
C_{n 1} & \cdots & C_{n n} & 1 & R_{n} \\
1 & \cdots & 1 & 0 & 0 \\
R_{1} & \cdots & R_{n} & 0 & 0
\end{array}\right)\left(\begin{array}{c}
\lambda_{1} \\
\vdots \\
\lambda_{n} \\
\mu_{0} \\
\mu_{1}
\end{array}\right)=\left(\begin{array}{c}
C_{10} \\
\vdots \\
C_{n 0} \\
1 \\
R_{0}
\end{array}\right)
$$

where $C_{i j}$ is the covariance of $Z(\mathbf{x}), C\left(\mathbf{x}_{i}-\mathbf{x}_{j}\right)=E\left\{\left(Z\left(\mathbf{x}_{i}\right)-m\left(\mathbf{x}_{i}\right)\right)\left(Z\left(\mathbf{x}_{j}\right)-\right.\right.$ $\left.\left.m\left(\mathbf{x}_{j}\right)\right)\right\}$ between locations $\mathbf{x}_{i}$ and $\mathbf{x}_{j}, R_{i}$ is the value of the drift $R$ at location $\mathbf{x}_{i}$, and $\mu_{0}$ and $\mu_{1}$ are Lagrange parameters, by-products of the constrained optimization that leads to Eq. (3).

In this work, the expression of the mean as a function of the drift is modified as

$$
m(\mathbf{x})=b \cdot R(\mathbf{x})
$$

to force the mean rainfall to be equal to zero when the radar estimate is zero. With this modification, the expression for the estimate remains Eq. (2) but the weights are the solution of the system

$$
\left(\begin{array}{cccc}
C_{11} & \cdots & C_{1 n} & R_{1} \\
\vdots & \ddots & \vdots & \vdots \\
C_{n 1} & \cdots & C_{n n} & R_{n} \\
R_{1} & \cdots & R_{n} & 0
\end{array}\right)\left(\begin{array}{c}
\lambda_{1} \\
\vdots \\
\lambda_{n} \\
\mu_{1}
\end{array}\right)=\left(\begin{array}{c}
C_{10} \\
\vdots \\
C_{n 0} \\
R_{0}
\end{array}\right)
$$


The only difference between Eqs. (3) and (5) is that, in the latter, the equation with the constrain that the sum of the weights must add up to one has disappeared. The resulting estimate $z_{K E D}^{*}\left(\mathbf{x}_{0}\right)$ can still be negative - because kriging is a nonconvex estimator and enforcing that the mean rainfall is zero when the radar is zero is not enough to make the kriging estimates positive. When this happens, the estimated value is set to zero.

The work by Velasco-Forero et al. (2009) uses the approach as described above to estimate rainfall from rain gauge and radar data accumulated over the same time interval. Since this estimation is two-dimensional, it will be referred to as KED2D.

The main innovation introduced by Velasco-Forero et al. (2009) was the automatic calculation of the covariance function using a non-stationary model for the mean. This automatic calculation is based on the work by Yao and Journel (1998) with the following considerations:

(i) It is decided that the covariance of rainfall is the same as the covariance of radar rainfall estimates. Since radar data are exhaustively sampled, it is quick and simple to compute an exhaustive experimental covariance.

(ii) The radar values are also modeled as non-stationary on the mean. Therefore, there is a need to get an estimate of the radar mean to compute the radar covariance. This estimate is obtained by ordinary kriging of the radar values at rain gauge locations. The covariance used in this ordinary kriging step is computed automatically using Yao and Journel's method, assuming stationarity on the mean. This stationary covariance is only a preliminary covariance used to obtain a smooth field for the radar mean.

(iii) Once this estimate of the radar mean is known, an experimental covariance map of radar is calculated, accounting for the non-stationarity on the mean.

(iv) The resulting experimental covariance map - computed on an exhaustive map of radar values - will rarely be positive definite. The method by Yao and Journel is applied to transform it into a positive-definite covariance:

(a) The correction to render the covariance map into positive definite is done in the frequency domain. For this reason, the spatial covariance is transformed into a frequency spectrum by Fast Fourier Transform.

(b) In the frequency domain, the two conditions that the spectrum has to satisfy are that it is positive for all frequencies and that its integral equals the covariance at lag zero. These two conditions are achieved by clipping the negative values followed by a smoothing of the experimental spectrum until its integral equals the covariance at lag zero.

(c) The back transformation of the resulting spectrum is a licit covariance map, according to Bochner's theorem (Bochner 1949).

The upper left image in Fig. 1 shows the positive-definite covariance map obtained as explained above for the 10-minute accumulated rain between 14:20 and 14:30 on September 7, 2015.

Once a valid covariance function is available, the estimation of the rain map is performed by KED2D using the rain gauge data as the primary data and the radar data as the drift variable.

This approach takes into account anisotropies and is fully automatic; therefore, it can be updated every time step and be used for the real-time forecast of runoff from rainfall. 
Velasco-Forero et al. (2009) applied this approach one time step at a time using the information from a single time interval. However, considering that there must also be temporal correlation, especially at 10-minute intervals, an approach is proposed to incorporate the data from the previous time interval into the estimation at the current one.

2.2 Kriging with External Drift Accounting for the Current and the Previous Time Steps

Time is incorporated into the estimation process as an additional third dimension but using a covariance that is non-stationary in time. Also, the search neighborhood in the time dimension is constrained to one time step backward in time. Under these considerations, the expressions from the previous section remain valid, making the abstraction that now the estimation is three-dimensional instead of two-dimensional, and taking care of the non-stationarity when building the covariance matrices.

For clarity, new expressions for the kriging estimate and the kriging system are developed next in which the non-stationarity of the covariance is made explicit. The data set is partitioned in two subsets (one for each time step), and the covariance matrices in the left-hand side of the kriging system are partitioned into four submatrices

Consider the simple kriging estimate of rainfall at time step $t$ as a linear function of the observations at the current time step $t$ and at the previous one $t-1$

$$
\begin{aligned}
z_{S K}^{*}\left(\mathbf{x}_{0}, t\right)=m\left(\mathbf{x}_{0}, t\right)+\sum_{i=1}^{n\left(\mathbf{x}_{0}\right)}\left\{\lambda_{i, t}\left(\mathbf{x}_{0}, t\right)\left[z\left(\mathbf{x}_{i}, t\right)-m\left(\mathbf{x}_{i}, t\right)\right]+\right. \\
\left.+\lambda_{i, t-1}\left(\mathbf{x}_{0}, t-1\right)\left[z\left(\mathbf{x}_{i}, t-1\right)-m\left(\mathbf{x}_{i}, t-1\right)\right]\right\},
\end{aligned}
$$

where $z_{S K}^{*}\left(\mathbf{x}_{0}, t\right)$ and $m\left(\mathbf{x}_{0}, t\right)$ are, respectively, the simply kriging estimate and the mean value of rain at location $\mathbf{x}_{0}$ and time $t ; n\left(\mathbf{x}_{0}\right)$ is the number of data locations found within the search neighborhood; $\lambda_{i, t}\left(\mathbf{x}_{0}, t\right)$ and $\lambda_{i, t-1}\left(\mathbf{x}_{0}, t-1\right)$ are the weights for time steps $t$ and $t-1$, respectively; $z\left(\mathbf{x}_{i}, t\right)$ and $z\left(\mathbf{x}_{i}, t-1\right)$ are the rain gauge observations for the time steps $t$ and $t-1$, respectively; and $m\left(\mathbf{x}_{i}, t\right)$ and $m\left(\mathbf{x}_{i}, t-1\right)$ are the mean values of rainfall for the rain gauge at location $\mathbf{x}_{i}$ for the time steps $t$ and $t-1$, respectively. Kriging with external drift relaxes the simple-kriging need to know the non-stationary mean $m(\mathbf{x}, t)$ by postulating that the mean is proportional to a known drift function

$$
m(\mathbf{x}, t)=b R(\mathbf{x}, t)
$$

where $R(\mathbf{x}, t)$ is the drift function, which in this case is the radar rainfall estimate. Notice that, similarly as done for KED2D, the mean is forced to be zero when the radar is zero. 
Substituting (7) into (6), a new estimate is obtained

$$
\begin{aligned}
z_{K E D}^{*}\left(\mathbf{x}_{\mathbf{0}}, t\right)= & \sum_{i=1}^{n\left(\mathbf{x}_{\mathbf{0}}\right)}\left[\lambda_{i, t}\left(\mathbf{x}_{\mathbf{0}}, t\right) z\left(\mathbf{x}_{i}, t\right)+\lambda_{i, t-1}\left(\mathbf{x}_{\mathbf{0}}, t-1\right) z\left(\mathbf{x}_{i}, t-1\right)\right]+ \\
& +b\left[R\left(\mathbf{x}_{0}, t\right)-\sum_{i=1}^{n\left(\mathbf{x}_{\mathbf{0}}\right)} \lambda_{i, t} R\left(\mathbf{x}_{i}, t\right)-\sum_{i=1}^{n\left(\mathbf{x}_{\mathbf{0}}\right)} \lambda_{i, t-1} R\left(\mathbf{x}_{i}, t-1\right)\right],
\end{aligned}
$$

where the unknown coefficient $b$ can be filtered out imposing the following constraint

$$
\sum_{i=1}^{n\left(\mathbf{x}_{0}\right)} \lambda_{i, t} R\left(\mathbf{x}_{i}, t\right)+\lambda_{i, t-1} R\left(\mathbf{x}_{i}, t-1\right)=R\left(\mathbf{x}_{0}, t\right) .
$$

With this constrain enforced, the KED estimator results

$$
z_{K E D}^{*}\left(\mathbf{x}_{\mathbf{0}}, t\right)=\sum_{i=1}^{n\left(\mathbf{x}_{\mathbf{0}}\right)}\left[\lambda_{i, t}\left(\mathbf{x}_{\mathbf{0}}, t\right) z\left(\mathbf{x}_{i}, t\right)+\lambda_{i, t-1}\left(\mathbf{x}_{\mathbf{0}}, t-1\right) z\left(\mathbf{x}_{i}, t-1\right)\right],
$$

and the coefficients are obtained by minimizing the square of the estimation error subject to constrain (9), which results in the following system of equations

$$
\begin{cases}\sum_{j=1}^{n\left(\mathbf{x}_{\mathbf{0}}\right)} \lambda_{j t} C_{i j t t}+\lambda_{j(t-1)} C_{i j t(t-1)}+\mu R_{i t}=C_{i t} & \text { for } i=1 \ldots n\left(\mathbf{x}_{\mathbf{0}}\right) \\ \sum_{j=1}^{n\left(\mathbf{x}_{\mathbf{0}}\right)} \lambda_{j t} C_{i j(t-1) t}+\lambda_{j(t-1)} C_{i j(t-1)(t-1)}+\mu R_{i(t-1)}=C_{i(t-1)} & \text { for } i=1 \ldots n\left(\mathbf{x}_{\mathbf{0}}\right) \\ \sum_{j=1}^{n\left(\mathbf{x}_{\mathbf{0}}\right)} \lambda_{j t} R_{j t}+\lambda_{j(t-1)} R_{j(t-1)}=R_{0 t} & \end{cases}
$$

where $C_{i j t t}$ are the covariances for a separation lag $\mathbf{h}_{i j}=\left(\mathbf{x}_{i}-\mathbf{x}_{j}\right)$ at time step $t, C_{i j(t-1)(t-1)}$ are the covariances for the same spatial lag at time step $t-1$, $C_{i j t(t-1)}$ and $C_{i j(t-1) t}$ are the covariances for the same spatial lag and a time lag separation of one, and $\mu$ is a Lagrange parameter. The system can be written in matrix form as

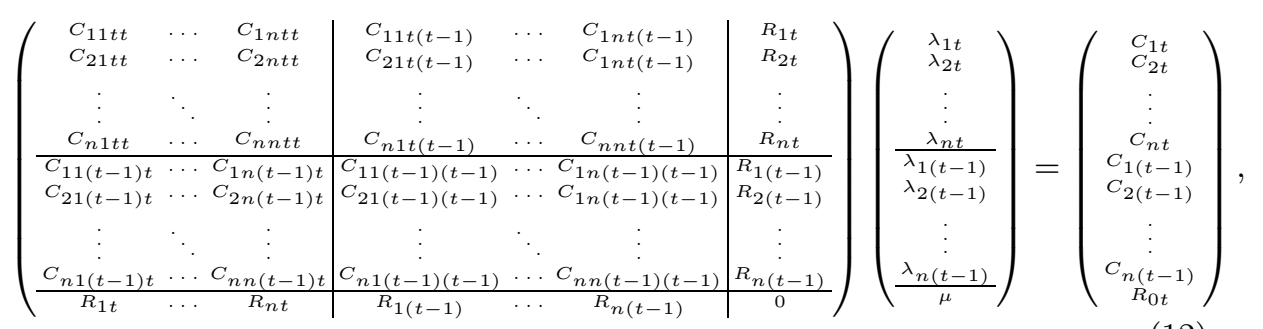

where $n$ is shorthand for $n\left(\mathbf{x}_{0}\right)$; the same system can be rewritten more compactly as

$$
\left(\begin{array}{lll}
\mathbf{C}_{t, t} & \mathbf{C}_{t, t-1} & \mathbf{R}_{t} \\
\mathbf{C}_{t-1, t} & \mathbf{C}_{t-1, t-1} & \mathbf{R}_{t-1} \\
\mathbf{R}_{t}^{T} & \mathbf{R}_{t-1}^{T} & 0
\end{array}\right)\left(\begin{array}{l}
\Lambda_{t} \\
\Lambda_{t-1} \\
\mu
\end{array}\right)=\left(\begin{array}{l}
\mathbf{C}_{t} \\
\mathbf{C}_{t-1} \\
R_{t}
\end{array}\right)
$$


This system of equations is a version of Eq. (5) expanded to account for two time steps, in which the covariances are computed using Yao and Journel's method, with time as a third dimension and considering all $t$ and $t-1$ observations as experimental data. In this compact expression, $\mathbf{C}_{t, t}$ is a matrix of dimensions $n\left(\mathbf{x}_{0}\right) \times n\left(\mathbf{x}_{0}\right)$ containing the covariances between pairs of data points at time $t$, and, similarly, $\mathbf{C}_{t-1, t-1}$ is a matrix of the same dimensions with the covariances computed at time $t-1$ (different from $\mathbf{C}_{t, t}$ because of the non-stationarity in time); $\mathbf{C}_{t, t-1}$ and $\mathbf{C}_{t-1, t}$ are the matrices of covariances between data points computed across time for a time lag of one, also of dimensions $n\left(\mathbf{x}_{0}\right) \times n\left(\mathbf{x}_{0}\right)$; these last two matrices, contrary to the previous two, are not necessarily symmetric since the correlation between location $\mathbf{x}_{i}$ at time $t$ and location $\mathbf{x}_{j}$ at time $t-1$ will, in general, be different from the correlation between location $\mathbf{x}_{i}$ at time $t-1$ and location $\mathbf{x}_{j}$ at time $t ; \mathbf{C}_{t}$ is a column vector of size $n\left(\mathbf{x}_{0}\right)$ with the covariances at time $t$ between the observation locations and location $\mathbf{x}_{0}$; and $\mathbf{C}_{t-1}$ is a vector of the same size with the covariances between the observation location at time $t-1$ and $\mathbf{x}_{0}$ at time $t ; \mathbf{R}_{t}$ is a column vector of size $n\left(\mathbf{x}_{0}\right)$ with the radar values at the rain gauge locations at time $t$, and $\mathbf{R}_{t-1}$ is another column vector of the same size with the radar values at the same locations at time $t-1$, the superscript $T$ indicates transpose; $\Lambda_{t}$ is a vector of size $n\left(\mathbf{x}_{0}\right)$ with the coefficients of the linear combination that apply to the data observed at time $t$, and, similarly, $\Lambda_{t-1}$ is a vector of the same size with the coefficients of the linear combination that apply to the data observed at time $t-1$; finally, $\mu$ is the Lagrange parameter and $R_{t}$ is the radar rainfall estimate at the estimation location, $\mathbf{x}_{0}$.

With this approach, the rainfall estimation presented here depends on:

(i) The rainfall data observed at $n\left(\mathbf{x}_{0}\right)$ rain gauges in time steps $t$ and $t-1$, $\left\{z\left(\mathbf{x}_{i}, t\right), z\left(\mathbf{x}_{i}, t-1\right), i=1, \ldots, n\left(\mathbf{x}_{0}\right)\right\}$.

(ii) The radar rainfall estimates at the same locations and time steps $\left\{R\left(\mathbf{x}_{i}, t\right), R\left(\mathbf{x}_{i}, t-\right.\right.$ $\left.1), i=1, \ldots, n\left(\mathbf{x}_{0}\right)\right\}$ plus the radar rainfall estimate at the location being estimated for time step $t, R\left(\mathbf{x}_{0}, t\right)$.

As mentioned previously, time is incorporated into the estimation process simply considering it as a third dimension and limiting the search neighborhood to one step backward in time. For this reason, this approach is referred to as KED3D.

For illustration purposes, Fig. 1 shows the covariance maps that should be used to fill the values in the kriging system. They have been computed using Yao and Journel's method with the radar data for the rainfall on September 7, 2015, during the two ten-minute intervals between 14:10 and 14:30. Recall that covariances are modeled as non-stationary in time. The position of the maps in the figure corresponds with the partitioning of the covariance matrix in Eq. (13).

\subsection{Accounting for the Motion of the Rainfall Field}

The rainfall fields show an apparent motion, which is the result of the combined effect of the winds at some steering level and the systematic precipitation growth and dissipation (Germann et al. 2006). This motion suggests that working in storm coordinates (i.e., in a Lagrangian coordinate reference system moving with the precipitation field) will maximize the representativeness of rainfall observations from time step $t-1$ to estimate the rainfall at time $t$ (Zawadzki 1973). Working 
in storm coordinates requires removing the effect of the motion field between time steps $t-1$ and $t$ before the estimation process. An approach to do this is by finding the spatial locations where the rainfall observations from time $t-1$ would have moved at time $t$, had they displaced along the rainfall field. This approach has been implemented in the Nowcasting algorithm (Berenguer et al. 2011) and is used here. The tracking algorithm is conceptually similar to Tracking Radar Echoes by Correlation by Rinehart and Garvey (1978) and permits displacing the rainfall observations (both rain gauges and radar) from the positions they have at $t-1$ to those that they would have at time $t$ if they had moved with the storm.

This procedure (illustrated in Figs. 2, 3 and 4) is applied to the rainfall observations in time $t-1$ prior to the estimation of the covariances in Eq. (13). In Fig. 2 , two snapshots of radar rainfall estimates, ten minutes apart, are shown. These two snapshots serve to compute the velocity field shown in Fig. 3. This velocity field indicates how to displace the radar field and the rain gauges of time $t-1$ to their hypothetical positions had they moved with the rain. The displaced values are shown in Fig. 4.

\section{Application}

\subsection{Hydrological Setting}

The study area is located in Catalonia (NE Spain), where three main mountain ranges can be found, the Pyrenees, with peaks over 3000 masl, the pre-coastal line, with summits above 1000 masl, and the litoral range, with mountains about 500 masl. The Pyrenees run approximately East-West, and the other two ranges are more or less oriented NE-SW in parallel with the coastline. Each of these ranges acts as a barrier for the warm and humid air coming from the Mediterranean on the East, which favors the appearance of convective cells responsible for high-intensity rains. In this area, the 10-year return period daily precipitation commonly exceeds $100 \mathrm{~mm}$. Accumulated rainfall over $200 \mathrm{~mm}$ in 24 hours is observed somewhere in the Spanish Mediterranean coast at least once per year.

Radar observations were obtained from the Creu del Vent radar, a singlepolarization C-band radar belonging to the Meteorological Service of Catalonia located in the center of the study area. Radar observations were processed with the Integrated Tool for Hydrometeorological Forecasting (EHIMI) (Corral et al. 2009), which includes algorithms for quality control and quantitative precipitation estimation from radar reflectivity. In this work, 10-minute rainfall accumulations, with a $1 \mathrm{~km}$ by $1 \mathrm{~km}$ resolution, have been used. A detailed description of the processing of the radar data can be found in Berenguer et al. (2015).

The ground observations correspond to rain gauges from the Meteorological Service of Catalonia network and include about 200 tipping-bucket rain gauges. The original values reported by the rain gauges have been integrated in time to provide rainfall accumulations over 10-minute periods (in consonance with the radar data). 


\subsection{Case Studies}

The proposed methodology is applied to the four episodes listed in Table 1. All four episodes are of high intensity and with high rainfall accumulation values.

\begin{tabular}{cccccc}
\hline Ep. & Date & \# gauges & $30^{\prime}[\mathrm{mm}]$ & $24 \mathrm{~h}[\mathrm{~mm}]$ & Reference \\
\hline 1 & $09 / 07 / 2005$ & 203 & 44.6 & 87.9 & Bech et al. (2007) \\
& & & $(32.4)$ & $(89.2)$ & \\
\hline 2 & $09 / 13 / 2006$ & \multirow{2}{*}{187} & 57.6 & 216.0 & Mateo et al. (2009) \\
& & & $(41.0)$ & $(154.4)$ & \\
\hline 3 & $10 / 18 / 2006$ & \multirow{2}{*}{188} & 40.2 & 93.6 & Aran et al. (2009) \\
& & & $(52.1)$ & $(114.3)$ & \\
\hline 4 & $11 / 02 / 2008$ & \multirow{2}{*}{186} & 40.0 & 130.2 & Bech et al. (2011) \\
& & & $(29.4)$ & $(88.2)$ & \\
\hline
\end{tabular}

Table 1 Episode, number of rain gauges, maximum 30-minute and maximum 24-h rainfall as observed in the rain gauges and in the radar (in parenthesis). The last column gives a reference for the reader interested in further details of the event.

\section{Results}

Episode 2 is described in detail, while only summary statistics will be given for the other three episodes. Episode 2 is the one with the largest 24-hour accumulation. It occurred on September 13, 2006, between 01:00 and 24:00 UTC. Estimates of rainfall are computed every ten minutes and then integrated for the entire episode. Estimates at a given time $t$ are obtained using four approaches: (i) the rainfall estimate is the radar rainfall estimate, (ii) the rainfall estimate is the KED value obtained using rain gauge and radar data at time $t$ (referred to as KED2D), (iii) the rainfall estimate is the KED value obtained using rain gauge and radar data at time $t$ and rain gauge and radar data at the previous 10-minute interval, without accounting for the motion of the rainfall field (referred to as KED3Dnm), and (iv) the rainfall estimate is the KED estimate obtained using rain gauge and radar data at time $t$ and rain gauge and radar data at the previous time step, accounting for the motion of the rainfall field (referred to as KED3D). Fig. 5 shows the total rainfall estimate by the four approaches.

The performance of each approach is evaluated by cross-validation. A rain gauge is removed from the data set; then, rainfall is estimated at the location of the removed rain gauge from the remaining data. This process is repeated for all rain gauge locations, at 10-minute intervals, to end with rainfall estimates at all rain gauge locations and time intervals. These estimates are compared with the (true) observed values to discern which approach performs best. Fig. 6 shows four scattergrams of total accumulation at rain gauge locations, one for each approach, radar data alone, KED2D, KED3Dnm, and KED3D. The horizontal axes are for the (true) values observed at the rain gauges, and the vertical axes are for the estimated values by each approach. It can be observed that the KED estimates are always better than the radar estimates and that KED3D is marginally better 
than KED2D and KED3Dnm. Fig. 7 shows the same results but for all 10-minute accumulated rainfall estimates during the entire episode. Similar conclusions can be reached, all the kriging estimates outperform the radar rainfall estimates, and KED3D is slightly better than KED2D and KED3Dnm.

This cross-validation analysis has been performed in all four episodes. To summarize the resulting scattergrams, the following statistics have been computed:

(i) The ratio between the average of all estimations and the average of all observations

$$
\mathrm{R} / \mathrm{G}=\frac{m_{V_{e}}}{m_{V_{o}}} .
$$

The optimal value is 1 .

(ii) The Pearson coefficient of linear correlation

$$
\text { corr }=\frac{\frac{1}{n} \sum_{i=1}^{n}\left(V_{o_{i}}-m_{V_{o}}\right)\left(V_{e_{i}}-m_{V_{e}}\right)}{\sigma_{V_{o}} \sigma_{V_{e}}} .
$$

The optimal value is 1 .

(iii) The Nash-Sutcliffe efficiency

$$
\mathrm{NS}=1-\frac{\sum_{i=1}^{n}\left(V_{o_{i}}-V_{e_{i}}\right)^{2}}{\sum_{i=1}^{n}\left(V_{o_{i}}-m_{V_{o}}\right)^{2}} .
$$

The optimal value is 1 .

(iv) The root mean square error

$$
\mathrm{RMSE}=\sqrt{\frac{1}{n} \sum_{i=1}^{n}\left(V_{e_{i}}-V_{o_{i}}\right)^{2}} .
$$

The optimal value is $0 \mathrm{~mm}$.

(v) The standard deviation of the error $\epsilon_{i}=V_{e_{i}}-V_{o_{i}}$

$$
\text { Std dev }=\sqrt{\frac{1}{n-1} \sum_{i=1}^{n}\left(\epsilon_{i}-m_{\epsilon}\right)^{2}} .
$$

The optimal value of is $0 \mathrm{~mm}$.

In all the previous equations, $n$ is the number of rain gauges where crossvalidation was performed, $V_{o_{i}}$ is the observed value at rain gauge $i, V_{e_{i}}$ is the estimated value at rain gauge $i, m_{V_{o}}$ is the mean value of the $n$ observed rainfall values, $m_{V_{e}}$ is the mean value of the $n$ estimated values, $\sigma_{V_{o}}$ is the standard deviation of the observed values, $\sigma_{V_{e}}$ is the standard deviation of the estimated values and $m_{\epsilon}$ is the mean value of the error $\epsilon$. The summary statistics for the scattergrams of the cross-validation of the the total accumulation for all four episodes are shown in Table 2 and the summary statistics for the scattergrams of the crossvalidation of the 10-minute accumulations are shown in Table 3.

After the analysis of Tables 2 and 3, it is concluded what was apparent by merely looking at the scattergrams of Episode 2: that any of the radar-rain gauges blending approaches (KED2D, KED3Dnm, or KED3D) is better than using the radar alone. The results of the different KED approaches show that including 


\begin{tabular}{cccccc}
\hline & & Sep 7, 05 & Sep 13,06 & Oct 18, 06 & Nov 2, 08 \\
\hline \multirow{2}{*}{ R/G } & RADAR & 0.83 & 0.67 & 0.99 & 0.68 \\
$(-)$ & KED2D & 0.97 & 0.95 & 0.98 & 0.91 \\
& KED3Dnm & 0.98 & 0.96 & 1.01 & 0.91 \\
& KED3D & $\mathbf{1 . 0 1}$ & $\mathbf{0 . 9 8}$ & $\mathbf{1 . 0 1}$ & $\mathbf{0 . 9 4}$ \\
\hline \multirow{2}{*}{ corr } & RADAR & 0.75 & 0.87 & 0.82 & 0.79 \\
$(-)$ & KED2D & 0.86 & 0.89 & 0.89 & 0.74 \\
& KED3Dnm & 0.86 & 0.89 & 0.89 & 0.74 \\
& KED3D & $\mathbf{0 . 8 7}$ & $\mathbf{0 . 9 0}$ & $\mathbf{0 . 8 9}$ & $\mathbf{0 . 7 5}$ \\
\hline \multirow{2}{*}{ NS } & RADAR & 0.51 & 0.50 & 0.64 & 0.30 \\
$(-)$ & KED2D & 0.73 & 0.78 & 0.79 & 0.51 \\
& KED3Dnm & 0.73 & 0.78 & 0.80 & 0.52 \\
\hline \multirow{2}{*}{ KSE } & RED3D & $\mathbf{0 . 7 4}$ & $\mathbf{0 . 8 1}$ & $\mathbf{0 . 8 0}$ & $\mathbf{0 . 5 6}$ \\
$(\mathrm{mm})$ & KED2D & 13.20 & 26.68 & 10.92 & 15.47 \\
& KED3Dnm & 9.78 & 17.77 & 8.33 & 12.89 \\
& KED3D & $\mathbf{9 . 4 9}$ & $\mathbf{1 6 . 3 2}$ & $\mathbf{8 . 2 0}$ & $\mathbf{1 2 . 3 4}$ \\
\hline \multirow{2}{*}{ Std dev } & RADAR & 12.49 & 20.83 & 10.95 & 11.47 \\
$(\mathrm{~mm})$ & KED2D & 9.78 & 17.64 & 8.34 & 12.58 \\
& KED3Dnm & 9.71 & 17.66 & 8.24 & 12.61 \\
& KED3D & $\mathbf{9 . 5 1}$ & $\mathbf{1 6 . 3 4}$ & $\mathbf{8 . 2 1}$ & $\mathbf{1 2 . 2 3}$ \\
\hline
\end{tabular}

Table 2 Summary statistics of the cross-validation scattergrams for total rainfall accumulation

the rainfall data from the previous time step and accounting for the motion of the rainfall field improves the scores systematically for all the events. Still, the improvement seems to be marginal (similarly as found by other authors who included the time component using different techniques, e.g., Sideris et al., 2014).

The proposed methodology is more realistic than previous attempts to include time in the estimation process since: (i) the covariances are computed and updated automatically for each time step, (ii) the expected value of rainfall is set to zero when the radar observation is zero, and (iii) the motion of the precipitation front is accounted for before doing any estimation.

The improvement obtained by the inclusion of the time component into the estimation process would be more significant when the method is applied in an area with a smaller density of rain gauges. The effect of the network density on the results of KED2D and KED3D shall be analyzed in future studies.

\section{Conclusions}

A new approach for the estimation of rainfall fields in real time has been proposed. The novelties of the approach include accounting for rain gauge and radar observations during two consecutive time steps and accounting for the motion of the rainfall to maximize the correlation across time steps. A moving coordinate system is used to displace the rainfall observations from time $t-1$ to time $t$ according to an estimated motion field. The automatic computation of the covariances, using 


\begin{tabular}{cccccc}
\hline & & Sep 7, 05 & Sep 13, 06 & Oct 18, 06 & Nov 2, 08 \\
\hline \multirow{3}{*}{ R/G } & RADAR & 0.83 & 0.67 & 0.99 & 0.68 \\
& KED2D & 0.97 & 0.95 & 0.98 & 0.91 \\
& KED3Dnm & 0.98 & 0.96 & 1.01 & 0.92 \\
& KED3D & $\mathbf{1 . 0 1}$ & $\mathbf{0 . 9 8}$ & $\mathbf{1 . 0 1}$ & $\mathbf{0 . 9 4}$ \\
\hline \multirow{3}{*}{ corr } & RADAR & 0.72 & 0.80 & 0.80 & 0.76 \\
& KED2D & 0.78 & 0.83 & 0.80 & 0.79 \\
& KED3Dnm & 0.79 & 0.83 & 0.81 & 0.79 \\
& KED3D & $\mathbf{0 . 8 0}$ & $\mathbf{0 . 8 4}$ & $\mathbf{0 . 8 1}$ & $\mathbf{0 . 8 0}$ \\
\hline \multirow{2}{*}{ NS } & RADAR & 0.48 & 0.58 & 0.63 & 0.53 \\
& KED2D & 0.60 & 0.67 & 0.63 & 0.60 \\
& KED3Dnm & 0.61 & 0.68 & 0.65 & 0.61 \\
RMSE & KED3D & $\mathbf{0 . 6 3}$ & $\mathbf{0 . 7 0}$ & $\mathbf{0 . 6 5}$ & $\mathbf{0 . 6 3}$ \\
$(\mathrm{mm})$ & RADAR & 0.56 & 0.91 & 0.62 & 0.45 \\
& KED2D & 0.49 & 0.82 & 0.61 & 0.41 \\
& KED3Dnm & 0.49 & 0.80 & 0.60 & 0.41 \\
Std dev & KED3D & $\mathbf{0 . 4 7}$ & $\mathbf{0 . 7 8}$ & $\mathbf{0 . 6 0}$ & $\mathbf{0 . 4 0}$ \\
$(\mathrm{mm})$ & RADAR & 0.56 & 0.91 & 0.62 & 0.44 \\
& KED2D & 0.49 & 0.81 & 0.61 & 0.41 \\
& KED3D & $\mathbf{0 . 4 7}$ & $\mathbf{0 . 7 8}$ & $\mathbf{0 . 6 0}$ & 0.40 \\
\hline
\end{tabular}

Table 3 Summary statistics of the cross-validation scattergrams for rainfall accumulation in 10-minute intervals

the same approach as in Velasco-Forero et al. (2009), allows its application in real time.

The results obtained for four rainfall events show that including the observations from the previous time step always has a positive effect (although the improvement seems to be small). Of the two approaches using the observations of the prior time step (KED3Dnm and KED3D), the one working in moving coordinates (i.e., accounting for the motion of the rainfall field) produces the best results.

\section{Acknowledgements}

This work has been done in the framework of the Spanish Project FFHazF (CGL201460700) and the EC H2020 project ANYWHERE (DRS-1-2015-700099). Thanks are due to the Meteorological Service of Catalonia for providing the radar and rain gauges data used here.

\section{References}

Aran, M., Amaro, J., Arús, J., Bech, J., Figuerola, F., Gayà, M., Vilaclara, E., 2009. Synoptic and mesoscale diagnosis of a tornado event in castellcir, catalonia, on 18th october 2006. Atmospheric Research 93, 147-160. 
Azimi-Zonooz, A., Krajewski, W., Bowles, D., Seo, D., 1989. Spatial rainfall estimation by linear and non-linear co-kriging of radar-rainfall and raingage data. Stochastic Hydrology and Hydraulics 3, 51-67.

Bech, J., Pascual, R., Rigo, T., Pineda, N., López, J., Arús, J., Gayá, M., 2007. An observational study of the 7 september 2005 barcelona tornado outbreak. Natural Hazards and Earth System Science 7, 129-139.

Bech, J., Pineda, N., Rigo, T., Aran, M., Amaro, J., Gayà, M., Arús, J., Montanyà, J., van der Velde, O., 2011. A mediterranean nocturnal heavy rainfall and tornadic event. part i: Overview, damage survey and radar analysis. Atmospheric research 100, 621-637.

Berenguer, M., Sempere-Torres, D., Hürlimann, M., 2015. Debris-flow forecasting at regional scale by combining susceptibility mapping and radar rainfall. Natural Hazards and Earth System Sciences 15, 587-602.

Berenguer, M., Sempere-Torres, D., Pegram, G.G., 2011. Sbmcast-an ensemble nowcasting technique to assess the uncertainty in rainfall forecasts by lagrangian extrapolation. Journal of Hydrology 404, 226-240.

Bochner, S., 1949. Fourier transforms. Princeton University Press, London, 219pp.

Brown, P.E., Diggle, P.J., Lord, M.E., Young, P.C., 2001. Space-time calibration of radar rainfall data. Journal of the Royal Statistical Society Series C, vol. 50, 221-241.

Calheiros, R., Zawadzki, I., 1987. Reflectivity-rain rate relationships for radar hydrology in brazil. Journal of climate and applied meteorology 26, 118-132.

Chumchean, S., Seed, A., Sharma, A., 2006. Correcting of real-time radar rainfall bias using a kalman filtering approach. Journal of Hydrology 317, 123-137.

Corral, C., Velasco, D., Forcadell, D., Sempere-Torres, D., Velasco, E., 2009. Advances in radar-based flood warning systems. the ehimi system and the experience in the besòs flash-flood pilot basin .

Creutin, J., Delrieu, G., Lebel, T., 1988. Rain measurement by raingage-radar combination: a geostatistical approach. Journal of Atmospheric and oceanic technology 5, 102-115.

Delrieu, G., Annette, W., Brice, B., Dominique, F., Laurent, B., Pierre-Emmanuel, K., 2014. Geostatistical radar-raingauge merging: A novel method for the quantification of rain estimation accuracy. Advances in Water Resources 71, 110-124.

Deutsch, C., 1991. The relationship between universal kriging, kriging with an external drift and cokriging. SCRF Report 4.

Germann, U., Turner, B., Zawadzki, I., 2006. Predictability of precipitation from continental radar images. part iv: Limits to prediction. Journal of the Atmospheric Sciences 63, 20922108.

Goudenhoofdt, E., Delobbe, L., 2009. Evaluation of radar-gauge merging methods for quantitative precipitation estimates. Hydrology and Earth System Sciences 13, 195-203.

Harrold, T., Austin, P., 1974. The structure of precipitation systems - a review. J. Rech. Atmos 8, 41-57.

Hevesi, J.A., Istok, J.D., Flint, A.L., 1992a. Precipitation estimation in mountainous terrain using multivariate geostatistics. part i: structural analysis. Journal of applied meteorology $31,661-676$.

Hevesi, J.A., Istok, J.D., Flint, A.L., 1992b. Precipitation estimation in mountanious terrain using multivariate geostatistics. part ii: Isohyetal maps. Journal of applied meteorology $31,677-688$.

Jewell, S.A., Gaussiat, N., 2015. An assessment of kriging-based rain-gauge-radar merging techniques. Quarterly Journal of the Royal Meteorological Society 141, 2300-2313.

Journel, A.G., Rossi, M., 1989. When do we need a trend model in kriging? Mathematical Geology 21, 715-739.

Krajewski, W.F., 1987. Cokriging radar-rainfall and rain gage data. Journal of Geophysical Research: Atmospheres 92, 9571-9580.

Mateo, J., Ballart, D., Brucet, C., Aran, M., Bech, J., 2009. A study of a heavy rainfall event and a tornado outbreak during the passage of a squall line over catalonia. Atmospheric Research 93, 131-146.

Pulkkinen, S., Koistinen, J., Kuitunen, T., Harri, A.M., 2016. Probabilistic radar-gauge merging by multivariate spatiotemporal techniques. Journal of Hydrology 542, 662-678.

Rinehart, R., Garvey, E., 1978. Three-dimensional storm motion detection by conventional weather radar. Nature 273, 287.

Rosenfeld, D., Amitai, E., Wolff, D.B., 1995. Improved accuracy of radar wpmm estimated rainfall upon application of objective classification criteria. Journal of applied meteorology 
$34,212-223$.

Rosenfeld, D., Wolff, D.B., Amitai, E., 1994. The window probability matching method for rainfall measurements with radar. Journal of applied meteorology 33, 682-693.

Rosenfeld, D., Wolff, D.B., Atlas, D., 1993. General probability-matched relations between radar reflectivity and rain rate. Journal of applied meteorology 32, 50-72.

Sempere-Torres, D., Corral, C., Raso, J., Malgrat, P., 1999. Use of weather radar for combined sewer overflows monitoring and control. J. Environ. Eng. 125, 372-380.

Seo, D.J., 1998. Real-time estimation of rainfall fields using radar rainfall and rain gage data. Journal of Hydrology 208, $37-52$.

Seo, D.J., Krajewski, W.F., Bowles, D.S., 1990. Stochastic interpolation of rainfall data from rain gages and radar using cokriging: 1. design of experiments. Water Resources Research $26,469-477$.

Sideris, I., Gabella, M., Erdin, R., Germann, U., 2014. Real-time radar-rain-gauge merging using spatio-temporal co-kriging with external drift in the alpine terrain of switzerland. Quarterly Journal of the Royal Meteorological Society 140, 1097-1111.

Sinclair, S., Pegram, G., 2005. Combining radar and rain gauge rainfall estimates using conditional merging. Atmospheric Science Letters 6, 19-22.

Sun, X., Mein, R., Keenan, T., Elliott, J., 2000. Flood estimation using radar and raingauge data. Journal of Hydrology 239, $4-18$.

Todini, E., 2001. A bayesian technique for conditioning radar precipitation estimates to raingauge measurements. Hydrology and Earth System Sciences Discussions 5, 187-199.

Velasco-Forero, C.A., Sempere-Torres, D., Cassiraga, E.F., Gómez-Hernández, J.J., 2009. A non-parametric automatic blending methodology to estimate rainfall fields from rain gauge and radar data. Advances in Water Resources 32, 986-1002.

Wilson, J.W., Brandes, E.A., 1979. Radar measurement of rainfall-a summary. Bulletin of the American Meteorological Society 60, 1048-1060.

Yao, T., Journel, A.G., 1998. Automatic modeling of (cross) covariance tables using fast fourier transform. Mathematical Geology 30, 589-615.

Yoon, S.S., Bae, D.H., 2013. Optimal rainfall estimation by considering elevation in the han river basin, south korea. Journal of Applied Meteorology and Climatology 52, 802-818.

Zawadzki, I., 1973. Statistical properties of precipitation patterns. J. Appl. Meteor. 12, 459472 . 

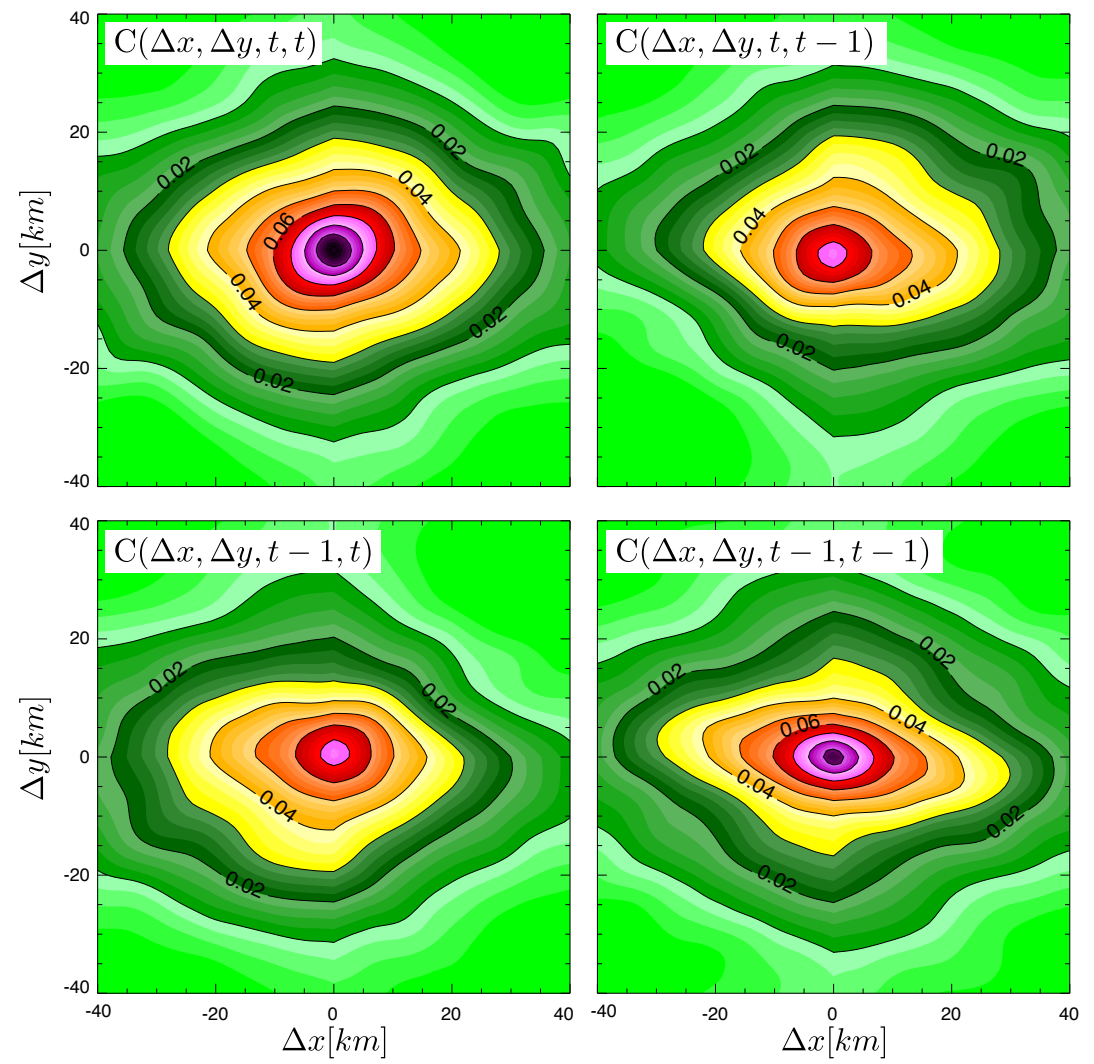

Fig. 1 Covariance maps for two time steps for the first episode analyzed 


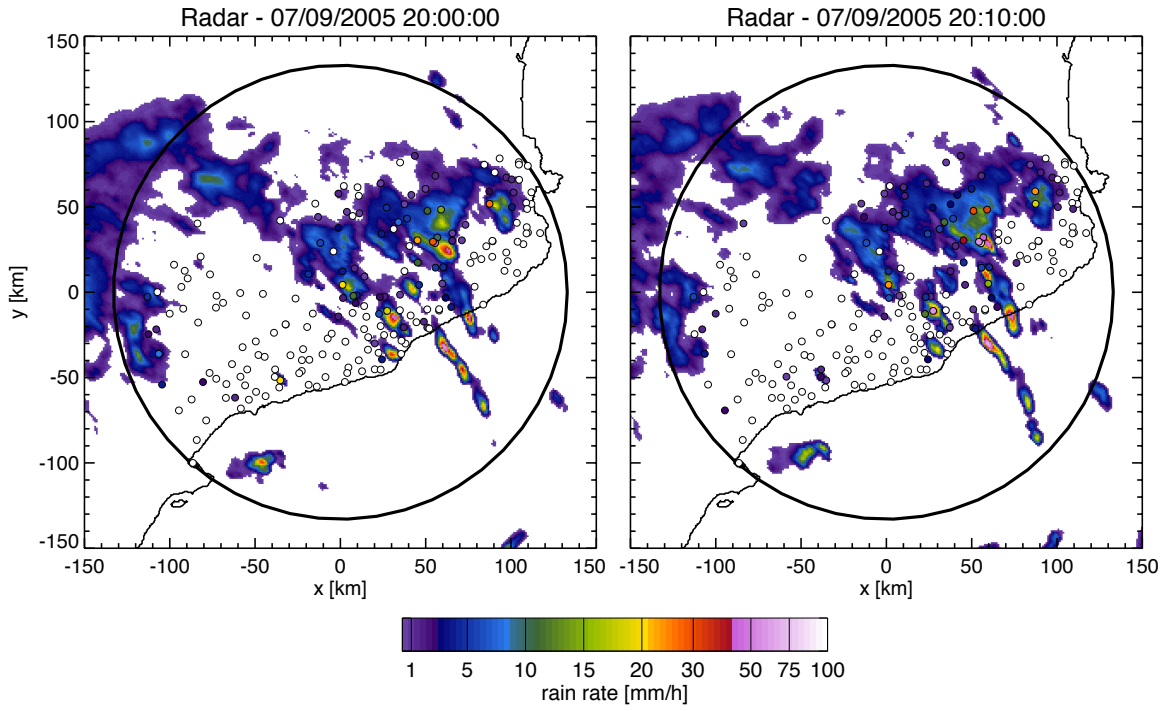

Fig. 2 Example of two radar images taken 10-minute apart. Rain gauge locations are shown, too

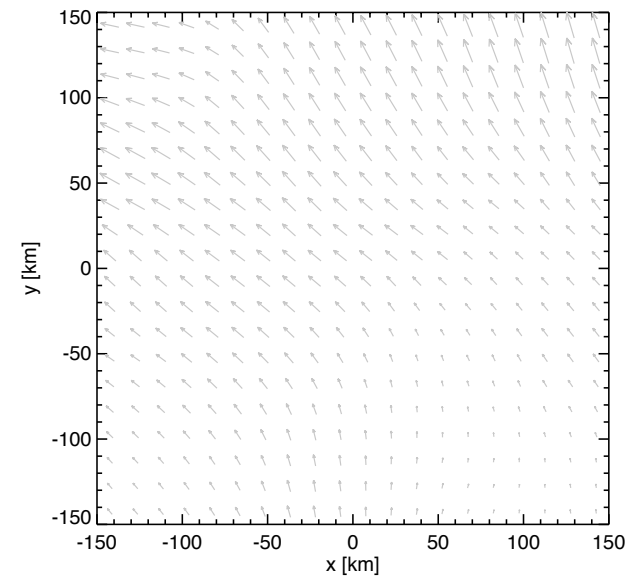

Fig. 3 Rainfall velocity field derived from the two radar images in the previous figure 


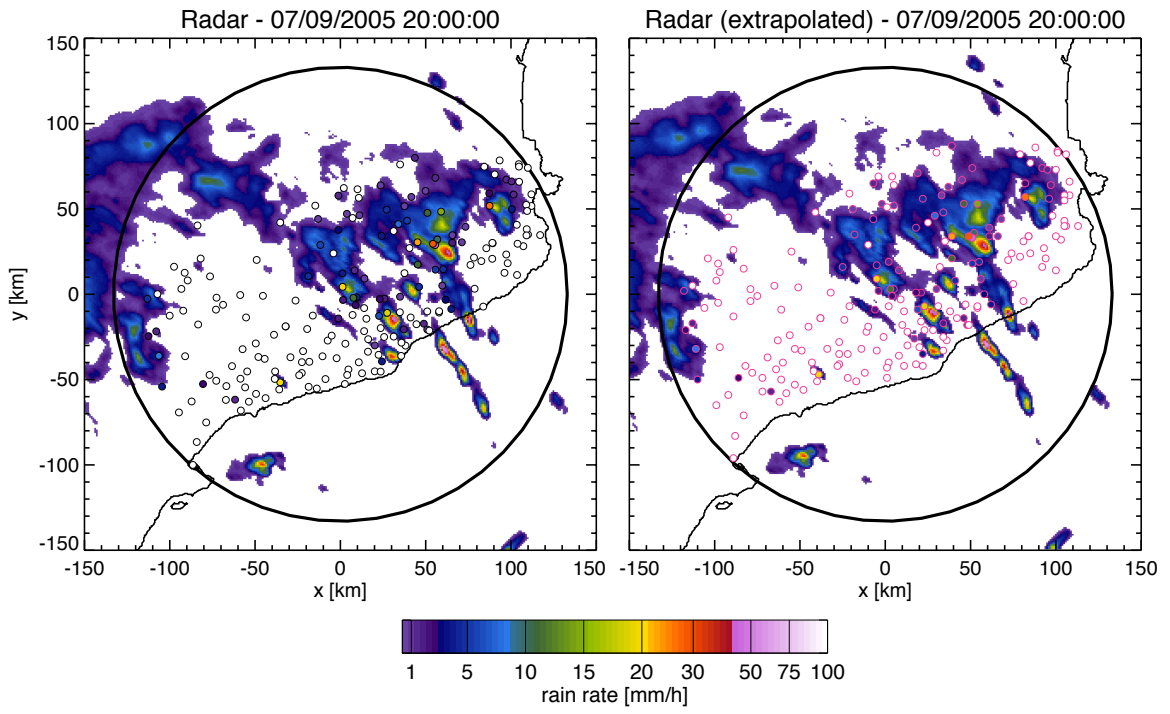

Fig. 4 Radar field at $t-1$ before (left) and after (right) displacement according to the velocity field of the previous figure. The displaced rain gauges are also shown (pink circles) 


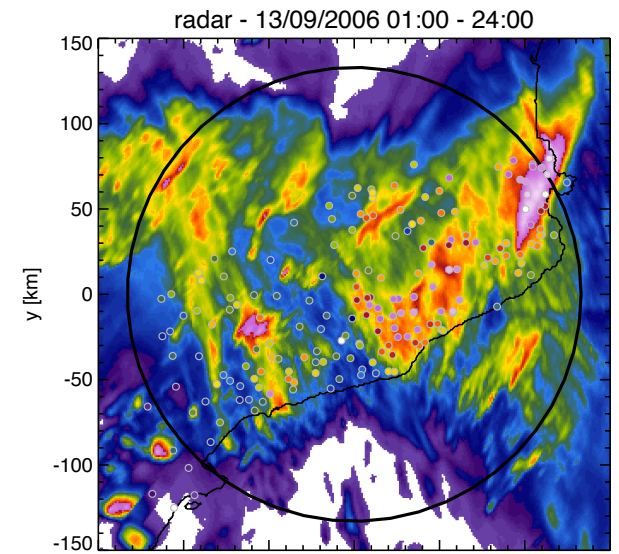

KED 3Dnm - 13/09/2006 01:00 - 24:00

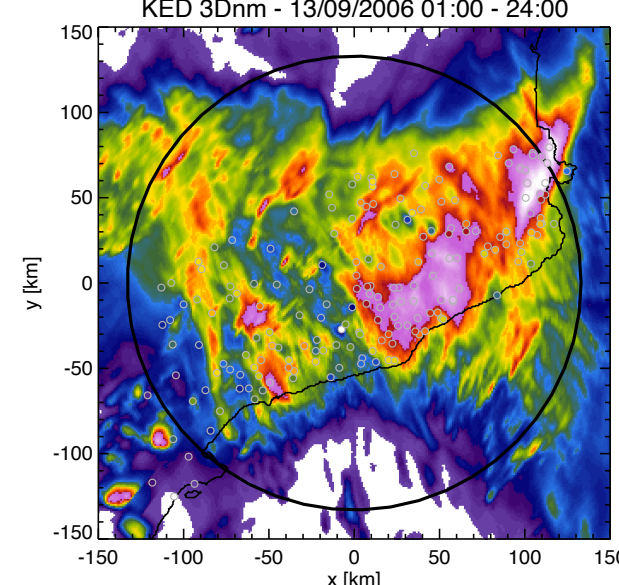

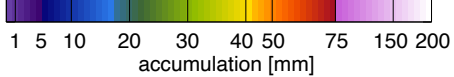

KED 2D - 13/09/2006 01:00 - 24:00

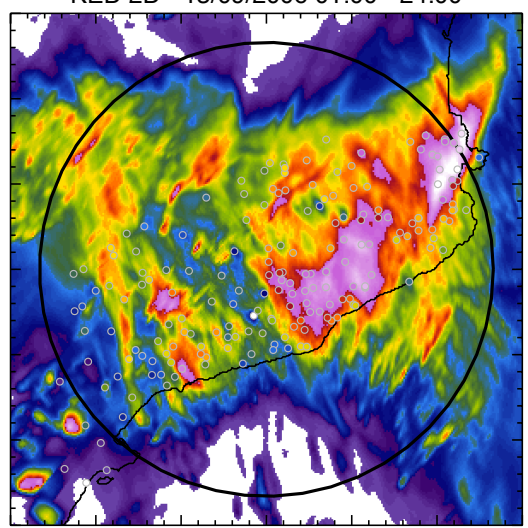

KED 3D - 13/09/2006 01:00 - 24:00

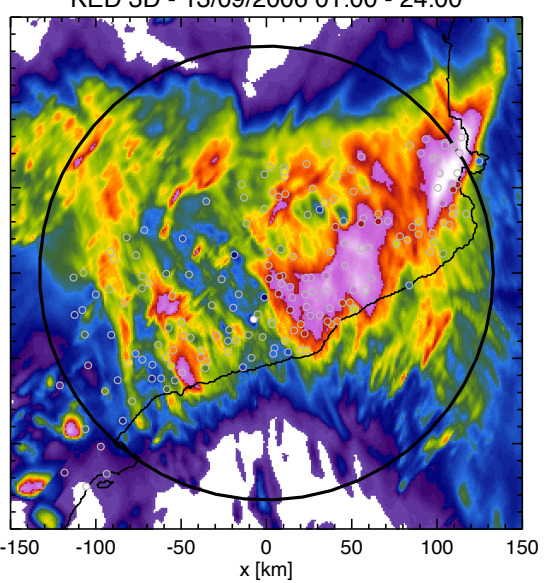

Fig. 5 Total rainfall as obtained by accumulating the 10-minute estimates using four approaches: radar alone, KED2D, KED3Dnm (without accounting for advection) and KED3D 

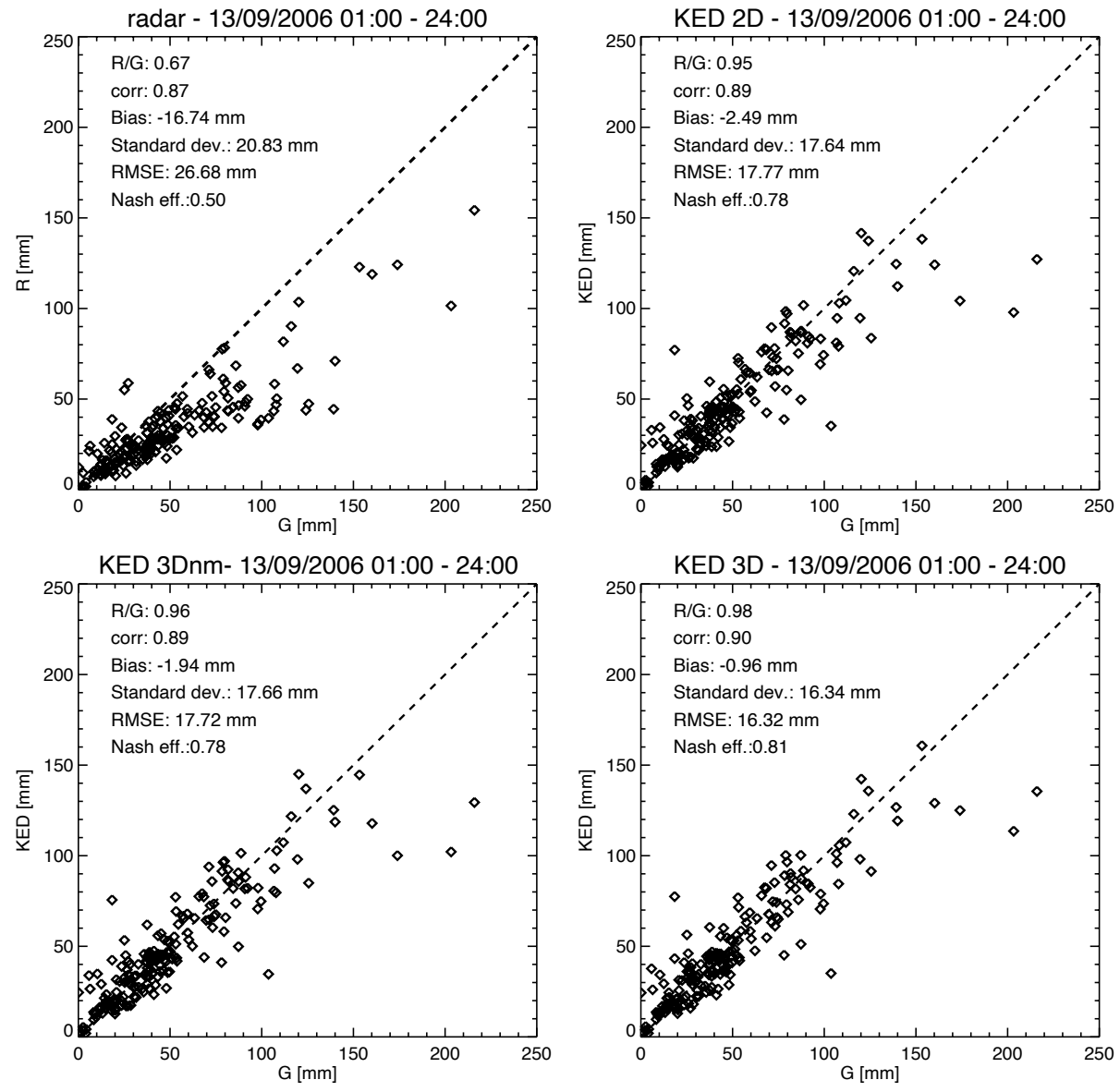

Fig. 6 Cross-validation scattergrams comparing the observed total accumulated rainfall at the 187 rain gauges vs. an estimated value obtained using four approaches: radar alone, KED2D, KED3Dnm and KED3D 

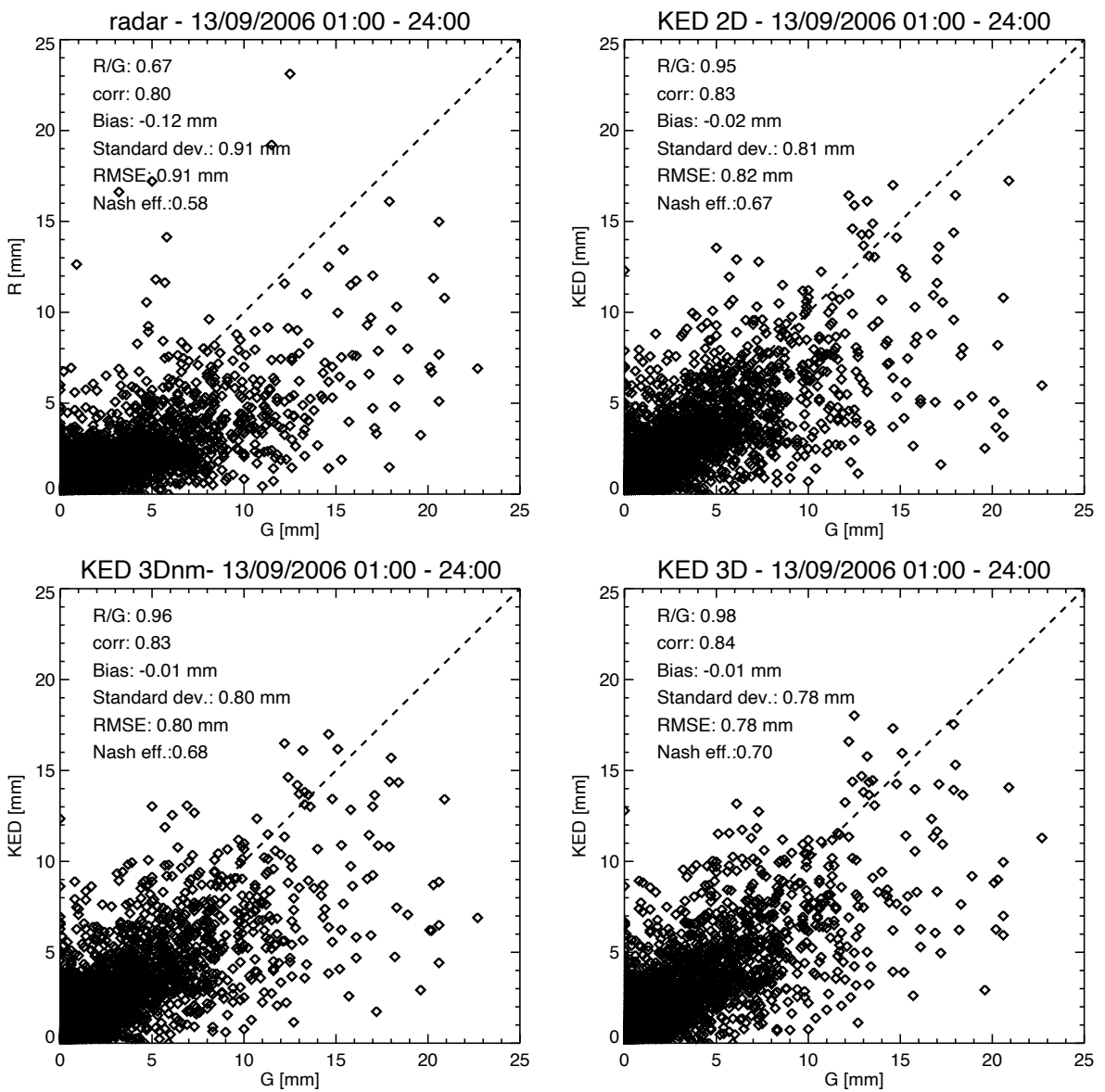

Fig. 7 Cross-validation scattergrams comparing the observed 10-minute accumulated rainfall at the 187 rain gauges vs. an estimated value obtained using four approaches: radar alone, KE2D, KE3Dnm and KE3D 